\title{
MR imaging of the spine in nonaccidental trauma
}

\author{
Giulio Zuccoli • Ashok Panigrahy
}

Received: 17 August 2009 /Revised: 30 September 2009 /Accepted: 12 November 2009/Published online: 5 December 2009

(C) Springer-Verlag 2009

An 8-week-old boy arrived intubated and was found to have a traumatic head injury, a coccygeal wound and multiple bruises. CT of the head showed a left parietal skull fracture with underlying subacute and chronic subdural hematomas. Ophthalmologic exam showed bilateral retinal hemorrhages. The hospital advocacy team suspected nonaccidental trauma after reviewing the history, physical exam and imaging findings. MRI of the brain showed bilateral frontal parietal cytotoxic edema and subdural subacute, chronic and subgaleal hematomas (not shown). MRI of the spine reveals a posterior intrathecal hematoma (probably subdural) with a fluid-fluid level consistent with blood product deposition (Fig. 1, arrows). Note also the diffuse swelling and hemorrhage in the subcutaneous tissues (Fig. 1, arrowheads) and ribs fracture with bone remodeling (Fig. 1, asterisk). The identification of the anatomic landmarks represented by the extradural fat (Fig. 2, arrow) and dura mater (Fig. 2, arrowheads) confirmed the intrathecal nature of the hematoma. The child was discharged home to protective care with the mother.

G. Zuccoli $(\bowtie) \cdot$ A. Panigrahy

Department of Pediatric Radiology,

Children's Hospital of Pittsburgh,

One Children's Hospital Drive 4401 Penn Ave.,

Pittsburgh, PA 15224, USA

e-mail: giulio.zuccoli@gmail.com

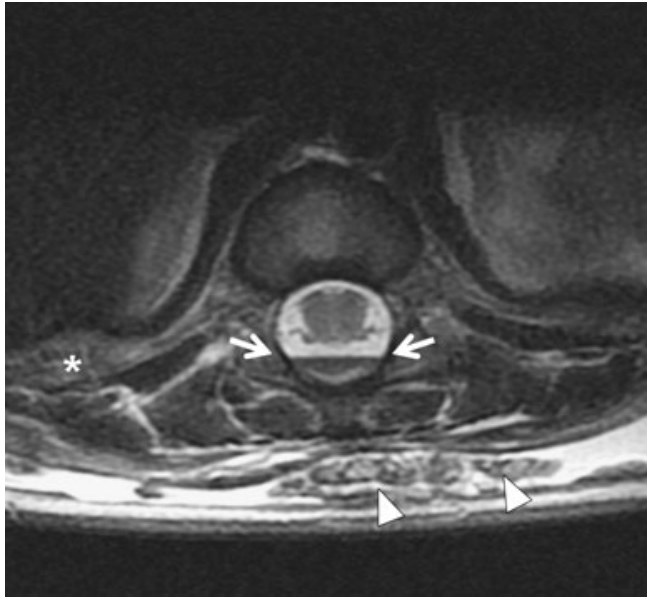

Fig. 1 MRI spine. T2-W axial image

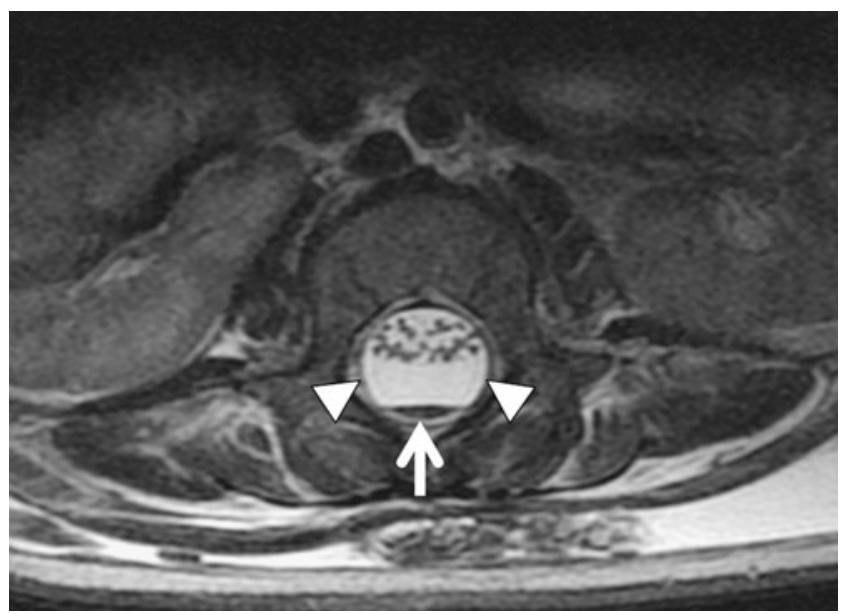

Fig. 2 MRI spine. T2-W axial image 